\title{
Hypothesis of the Hidden Multiverse Explains Dark Matter and Dark Energy
}

\author{
Alexander Alexandrovich Antonov ${ }^{1}$ \\ ${ }^{1}$ Research Center of Information Technologies “TELAN Electronics”, Kiev, Ukraine \\ Correspondence: Alexander Alexandrovich Antonov, Research Center of Information Technologies "TELAN \\ Electronics", Kiev, Ukraine. E-mail: telan@bk.ru
}

Received: January 19, 2016

Accepted: February 6, 2017 Online Published: February 16, 2017

doi:10.5539/apr.v9n2p30

URL: https://doi.org/10.5539/apr.v9n2p30

\begin{abstract}
There are currently a large number of Multiverse hypotheses, which are, however, non-verifiable, i.e. they can be neither confirmed nor refuted experimentally even in the distant future. In contrast, the hypothesis of the hidden Multiverse considered in the article is verifiable and therefore has a right to be called a theory. The theory uses the principle of physical reality of imaginary numbers discovered 500 years ago, including complex and hypercomplex numbers, as fundamental and proved by the author theoretically and experimentally. This principle has allowed revealing a number of serious mistakes in the special theory of relativity. An adjusted version of the special theory of relativity has been proposed and the theory of the hidden Multiverse has been developed on its basis. The Multiverse has been referred to as hidden, because parallel universes it contains are mutually invisible. The nature of their invisibility is explained in the article. It is shown that dark matter and dark energy are other universes of the hidden Multiverse apart from ours. Analysis of data from WMAP and Planck spacecrafts has shown that the hidden Multiverse has quaternion structure comprising four pairs of universes and antiverses (i.e., four pairs of matter and antimatter).
\end{abstract}

Keywords: physical reality of imaginary numbers, special theory of relativity, dark matter, dark energy, Multiverse

\section{Introduction}

Sensational report about the OPERA experiment (Adam et al., 2011) carried out at the Large Hadron Collider was published on September 22, 2011. As a result, superluminal neutrinos were allegedly registered. The experiment thereby refuted the principle of light speed non-exceedance in the special theory of relativity (STR) (Einstein, 1920; Hawking \& Penrose, 2010) and proved physical reality of imaginary numbers discovered 500 years ago. In short time after that, a great amount of scientific publications appeared. They were mainly devoted to the search for possible evidence of the fallacy of such a result. Some publications on the subject even argued that the results were so incredible that should they appear to be true, they would have deserved the Nobel Prize.

On the March 29, 2012 ICARUS experiment report (Antonello al., 2012) was published. It disproved the results of OPERA experiment. ICARUS experiment didn't thereby prove correctness of the principle of light speed non-exceedance.

However, the situation described raises questions:

- Why such complex, costly and long-term experiments as OPERA, MINOS (Adamson et al., 2007) and others, should be carried out to refute the principle of light speed non-exceedance in the STR and to prove physical reality of imaginary numbers, if much simpler experiments, which demonstratively proved physical reality of imaginary numbers, had already been conducted and published (Antonov, 2008; 2009; 2010a; 2010b; 2010c) by then? Besides, since these alternative experiments referred to the study of oscillation processes in linear electric circuits, there, consequently, was every possibility to repeat and, therefore, verify them in any electric or electronic laboratory. That fact proved their reliability.

- However, if such great efforts were made to refute the OPERA experiment, why then alternative experiments having resolved the same physically important issue that was the objective of the OPERA experiment, has not been yet commented in the preceding years? Why did interest in this issue arouse only for six months: between the dates of publication of the OPERA and ICARUS experiments' results, and was lost in subsequent years? It's because physical community seemed to have no need in the truth. It needed refutation 
of the possibility to solve, one day, the issue of physical reality of imaginary numbers and the principle of light speed non-exceedance, rather than its verification. The results of the alternative experiments appeared to be irrefutable and physical community, therefore, lost interest in them.

Therefore, the article:

- $\quad$ shows that the existing version of the STR is incorrect and still should be adjusted;

- $\quad$ shows what issues that haven't been yet solved in terms of the existing version of the STR, have been solved in terms of the adjusted version of the STR.

\section{Physical Reality of Imaginary Numbers}

At first, we show how physical reality of imaginary numbers has been proved.

\subsection{The First Proof}

The simplest of the three proofs (Antonov, 2015a; 2015b; 2016a) is a proof using Ohm's law discovered in 1826 (Ohm, 2014). However, Ohm's law was discovered, when there were no electrical appliances. Therefore, it has been discovered with respect to the electric DC-circuits. With regard to electric AC-circuits Ohm's law was formulated by Charles Proteus Steinmetz in 1897 (Steinmetz, 2010). According to the interpretation of Ohm's law proposed by him, not only resistors, but also inductors and capacitors have electrical resistance. Besides, in contrast to the resistance of resistors, measured by real numbers, resistance of capacitors and inductors are measured by heteropolar imaginary numbers. Therefore, the resistance itself is also often called imaginary. Moreover, they are also often understood as imaginary, i.e., as physically nonexistent.

However, this is wrong. If resistance of inductors and capacitors was physically nonexistent, the magnitude of electric current flowing through the LCR-circuit while changing the frequency of the sinusoidal voltage applied to it should not be changed. However, it does change, which proves physical reality of imaginary resistance of capacitors and inductors, and thus physical reality of imaginary numbers, and allows refutation of the principle of light speed non-exceedance.

Furthermore, if imaginary resistance of capacitors and inductors were physically nonexistent, there would be no resonance phenomenon in electric LCR-circuits, which was discovered by Galileo di Vincento Bonaiuti de'Galilei (Frova \& Marenzana, 2006) in 1602. Such sciences as radio engineering, telecommunications, radiolocation and others also wouldn't exist.

\subsection{The Second Proof}

Another proof is based on an accurate analysis of resonance processes in the electric LCR-circuits (Antonov, $1987 ; 2008 ; 2009 ; 2010 \mathrm{a} ; 2010 \mathrm{~b} ; 2015 \mathrm{c}$ ), as against the approximate analysis presented in all textbooks, which was carried out under dominant influence of the STR. Therefore, all textbooks still assert that resonance is possible at real frequencies and has the following attributes:

- at resonant frequency the module of complex resistance (or, in general, of immittance function) of the electric LCR-circuit concerned takes on extreme value;

- at resonant frequency the imaginary component of the complex resistance (or, in general, of immittance function) of the electric LCR-circuit concerned takes on zero value;

- resonant frequency is equal to the frequency of free oscillations.

In fact, these resonance attributes are observed only in electric LC-circuits, representing limited practical interest. As applied to the electric LCR-circuits, none of the resonance attributes is verified experimentally (these attributes are rather approximately correspondent to reality).

As accurate analysis of resonance processes in these circuits has shown, resonance actually takes place at complex frequencies, which proves physical reality of complex (and therefore imaginary) numbers.

Existence of resonance at complex frequencies is even patented (Antonov \& Bazhev, 1970).

\subsection{The Third Proof}

The last proof (Antonov, 2010c; 2016c) is probably the most convincing, as it is confirmed by experiments set up by nature, i.e. by shock oscillations in the form of tsunami, sound of stringed musical instruments, and even a kid's swing being pushed by parents to get a swinging motion. Their existence certainly cannot be refuted by ICARUS experiment.

Indeed, any processes are mathematically described by differential equations, and oscillation transients, the particular case of which are shock oscillations mentioned above, are determined by appropriate characteristic 
algebraic equations. Oscillation transient corresponds only to solutions in the form of pairs of complex conjugate numbers. However, if these numbers were physically nonexistent, characteristic equation should be solved on the set of real numbers and one we would have to assert that oscillation transients should not ever exist. However, they do exist. And the fact of their existence proves physical reality of complex (and, therefore, imaginary) numbers.

Other proofs of physical reality of imaginary numbers have also been published (Lyahov \& Nechshadim, 2001; Spenkov, 2014).

\section{Physical Nature of Imaginary Numbers: Adjustment of the STR}

Nature is unified and consistent. Otherwise, it couldn't exist. Therefore, Science as a whole should also be as such. Some scientific disciplines, the existence of which can only be explained by limited intellectual capacity of people, should also be mutually consistent. If this condition is sometimes not satisfied (for example, inconsistency between the theory of relativity and quantum physics), at least one of the theories would be incorrect.

Consequently, the principle of the physical reality of imaginary numbers proved in the theory of electric circuits is generally scientific. Of course, it is very unusual. However, this is a good place to mention Isaac Newton's opinion on this occasion: "No great discovery was ever made without a bold guess". Therefore, scientific theories and hypothesis in all scientific disciplines such as theory of relativity, quantum physics, optics, theory of electric circuits and others should be adjusted in accordance with this principle.

As shown above, imaginary resistance and conductance of capacitors and inductors are imaginary physical entities that actually exist in the theory of electric circuits.

We now show what physical sense physical entities have in the STR (Antonov, 2014a). For this purpose, some relativistic formulas should be considered. For example

$$
\begin{gathered}
m=\frac{m_{0}}{\sqrt{1-(v / c)^{2}}} \\
\Delta t=\Delta t_{0} \sqrt{1-(v / c)^{2}}
\end{gathered}
$$

where $i=\sqrt{-1}$ is the imaginary unit;

$\boldsymbol{m}_{\boldsymbol{0}}$ is the rest mass;

$\boldsymbol{m}$ is the relativistic mass;

$\Delta t_{0}$ is the rest time;

$\Delta t$ is the relativistic time;

$v$ is the velocity of a moving physical entity;

$c$ is the speed of light.

As can be seen, at subluminal speeds, when $\boldsymbol{v}<\boldsymbol{c}$, relativistic mass $\boldsymbol{m}$ and relativistic time $\boldsymbol{\Delta} \boldsymbol{t}$ are measured by real numbers, whereas at superluminal speeds, when $\boldsymbol{v}>\boldsymbol{c}$, these values are measured by imaginary numbers. STR authors couldn't understand what the sense of these physical entities was. Therefore, to avoid the necessity of explaining it or acknowledging their inability to explain, they needed to use the principle of light speed non-exceedance, which was actually postulated (because it wasn't proved anywhere) (Antonov, 2014b). The principle, being justified using the assertion about the impossibility to overcome light speed barrier, is easily refuted, even at the mundane level. Indeed, inability, let us say, to get into the adjacent room through the wall does not mean that there is no way to get there through the door. It neither means that the room and its contents do not exist.

However, the sense of relativistic formulas at $v>c$ should be explained now, as physical reality of imaginary numbers has been already proved. Imaginary quantities, i.e. relativistic mass, relativistic time and other imaginary physical entities have the following explanation in the STR. Physical bodies, such as for example tachyons (Tanaka, 1960; Feinberg, 1967), are invisible to us (Antonov, 2015d) at superluminal speeds, because they are, by definition, outside the event horizon. Therefore, they are in another universe, rather than in ours. This 'another' universe can be for clarity referred to as a tachyon, whereas our universe can be called a tardyon (as derived from the name of elementary particles, moving at sub-light speed). It follows from the above that we live in a Multiverse, which we have called a hidden Multiverse due to its invisibility (Antonov, 2011; 2012; 2015d; 2015e; 2015f; $2015 \mathrm{~g})$. 
According to the first postulate of the STR, physical, chemical and other laws in the hidden Multiverse have the same manifestations for the inhabitants of the tachyon universe, as they have for the inhabitants of our tardyon universe. However, as can be easily checked, relativistic formulas, including formulas (1) and (2), do not correspond to this condition. Therefore, they can be adjusted as follows:

$$
\begin{gathered}
m=\frac{(i)^{q} m_{0}}{\sqrt{1-(v / c-q)^{2}}}=\frac{(i)^{q} m_{0}}{\sqrt{1-(w / c)^{2}}} \\
\Delta t=(i)^{q} \Delta t_{0} \sqrt{1-(v / c-q)^{2}}=(i)^{q} \Delta t_{0} \sqrt{1-(w / c)^{2}}
\end{gathered}
$$

where $q=\lfloor v / c\rfloor$ is the discreet 'floor' function of argument $v / c$;

$\boldsymbol{w}=\boldsymbol{v}-\boldsymbol{q c}$ is the local velocity for each universe, which can take values only in the range $0 \leq \boldsymbol{w}<\boldsymbol{c} ;$

$\boldsymbol{v} \quad$ is the velocity measured from our universe;

c is the speed of light.

As shown in formulas (3) and (4), value $\boldsymbol{q}$ has appeared due to the fact that the velocity of a moving entity $\boldsymbol{v}$ can exceed the speed of light $\boldsymbol{c}$. Herewith, tardyon universe corresponds to the value $\boldsymbol{q}=\boldsymbol{0}$, and tachyon universe corresponds to the value $\boldsymbol{q}=\mathbf{1}$. But in formulas (3) and (4) the value $\boldsymbol{q}$ can be greater than one.

In this case, tardyon antiverse would correspond to the value $\boldsymbol{q}=\mathbf{2}$ (as $\boldsymbol{i}^{2}=-\mathbf{1}$ ), tachyon antiverse would correspond to the value $q=3$ (as $i^{3}=-i$ ), another tardyon universe would correspond to the value $q=4$ (as $\boldsymbol{i}^{4}=1$ ), another tachyon universe would correspond to the value $q=5$ (as $\boldsymbol{i}^{5}=\boldsymbol{i}$ ) and so on. In fact, such a space-time structure was predicted by Stephen William Hawking, who wrote: "Imaginary time is a new dimension, at right angles to ordinary, real time”. That is, it is permissible to assume that the structure of the hidden Multiverse is in the form of a screw collar. The example of such a structure, in which the total number of universes is twenty-four, is shown in Figure 1.

As can be seen, each universe in the structure of the hidden Multiverse corresponds to its integer $\boldsymbol{q}$ (like the number of flats in an apartment building). Therefore, if the value $\boldsymbol{q}$ in the formulas (3) and (4) is assumed to be the fourth spatial coordinate, different parallel universes (as they never intersect despite their immensity) would appear to exist in different dimensions. In this case it can be assumed that we live in a four-dimensional space (regardless of time).

In this multidimensional space different parallel universes slightly drift relative to each other all the time. Sometimes they touch each other in some spots (quite numerous) and even superficially penetrate into each other. Then, these places form some transition zones, which enable transition of small physical objects ranging from elementary particles to the living inhabitants of universes, from one parallel universe to another. However, such physical objects as planets, stars and galaxies cannot transit through such transition zones, since universes could be destabilized. Using existing terms, such transition zones can be referred to as portals or star gates. Besides, portals, as can be seen from their origin, are in no way identical to wormhole in the general theory of relativity.

Such portals exist on the Earth, i.e. on, above and below its surface, in the depths of lakes, seas and oceans. Most likely they are the so-called anomalous zones. According to the law of communicating vessels parameters of environment in areas before and beyond the portals should be almost identical. Therefore, transitions through the portals might be relatively safe, as, for example, safe is household electric network, if it is not touched. Should at least one portal between the Earth and space be opened, the Earth would have remained without its atmosphere and hydrosphere. This might supposedly have once happened on Mars.

Hence, according to the law of communicating vessels, mass-energy of different parallel universes of the hidden Multiverse has been substantially averaged over time. 


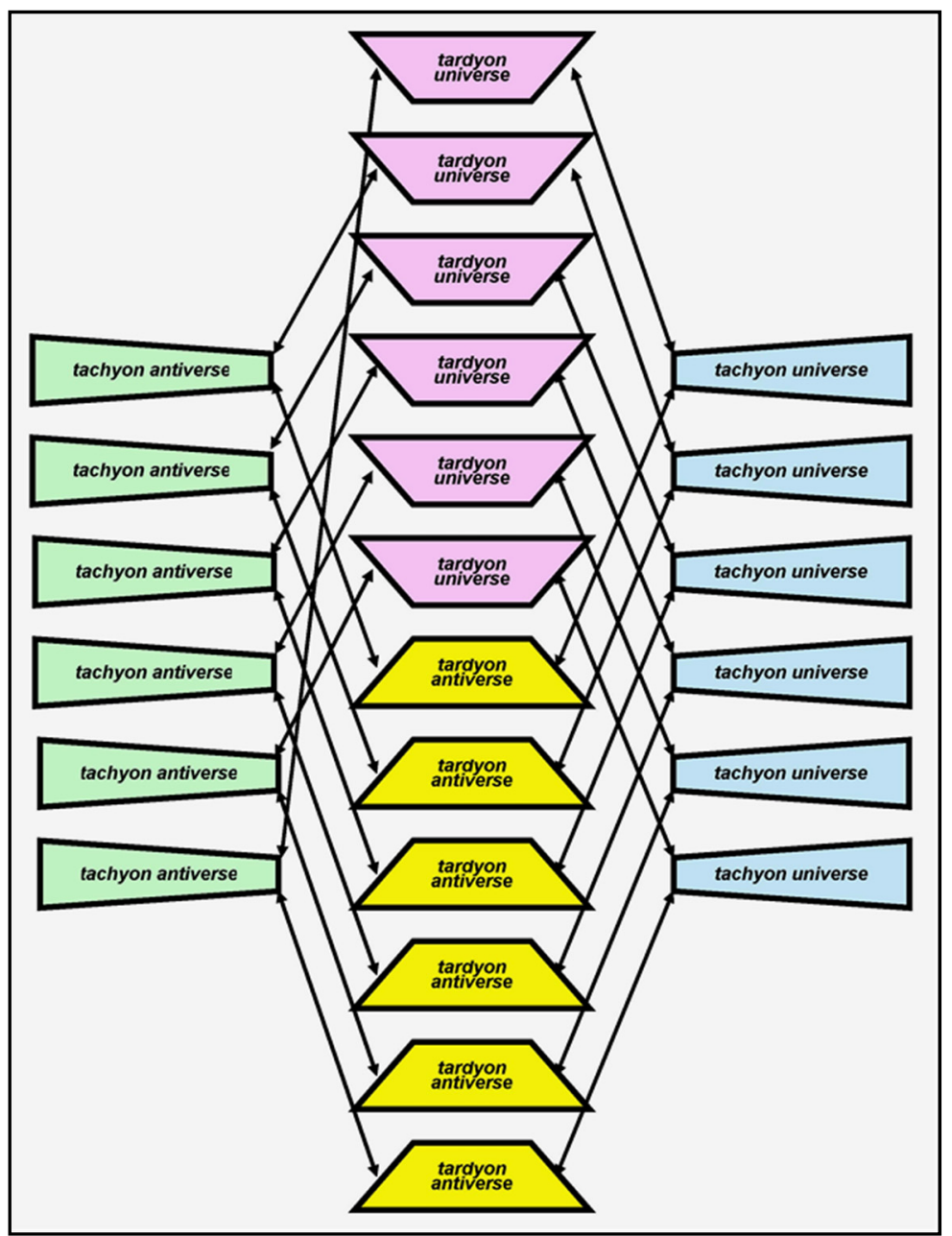

Figure 1. Possible structure of the hidden Multiverse, corresponding to the principle of physical reality of complex numbers

\section{Explanation of Dark Matter and Dark Energy}

Consideration of the hidden Multiverse can in no way be deemed completed without explaining the phenomenon of dark matter and dark energy (Freeman \& McNamara, 2006; Nicolson, 2007), to consideration of which we are now going to proceed to. Let's recall that these astrophysical objects of research are referred to as such, because they are invisible, i.e. neither emit nor reflect nor absorb light in any range of electromagnetic waves. Therefore, they were detected only due to gravitational lensing. They are called so also because they are absolutely incomprehensible. Thus, none of chemical elements existing in our universe as well as no subatomic particles have been detected in the composition of dark matter and dark energy. Their discovery has raise doubts about the modern understanding of the term 'matter'.

In fact, astrophysics themselves have created all these incomprehensibilities by incorrect statement of the task of finding explanation of the phenomenon, which should certainly correspond to the existing incorrect version of the special theory of relativity, stating that we live in a monoverse.

Therefore, Albert Einstein's remark is quite applicable to the current situation: "Insanity: doing the same thing over and over again and expecting different results".

If we change the statement of the task and seek an explanation for the phenomenon of dark matter and dark energy in the hidden Multiverse, explanation becomes obvious (Antonov, 2015h; 2015i; 2015k; 2016b):

- dark matter and dark energy correspond to other invisible parallel universes of the hidden Multiverse, except ours;

- of which parallel universes of the hidden Multiverse adjacent to our universe correspond to dark matter; 
- and the rest of parallel universes of the hidden Multiverse, which are more distant from us, correspond to dark energy;

- chemical composition of the contents of other parallel universes cannot be determined by tools that are in our universe.

Thus, the phenomenon of dark matter and dark energy discovered by astrophysicists proves physical existence of the hidden Multiverse and is essentially a different name for its other invisible parallel universes, except ours.

As can be seen, the proposed explanation corresponds to the Occam's razor criterion and therefore is the most plausible.

Emphasizing the great importance of explaining this phenomenon, Stephen William Hawking wrote: "The missing link in cosmology is the nature of dark matter and dark energy."

\section{Explanation of where Antimatter could be Found}

Antimatter is no less a mystery than the phenomenon of dark matter and dark energy (Alfvén, 1966; Frazer, 2004). Indeed, according to the big bang theory there are both matter and antimatter in the space. But where is antimatter? And if it actually exists somewhere, why then matter and antimatter didn't destroy each other soon after the big bang as a result of annihilation? That is, our universe even might not exist. But it (as well as people) does exist. According to the recent data it has existed for a long time, around $13,77 \pm 0,059$ billion years.

So how all this could be explained?

We have to admit that the phenomenon of antimatter cannot be explained within the hypothesis of monoverse which corresponds to the current erroneous version of the STR. In any case, no one could propose the explanation to this day.

Confucius wrote on the subject: "The hardest thing of all is to find a black cat in a dark room, especially if there is no cat."

However, the explanation is obvious within the proposed hypothesis of the hidden Multiverse. It is antiverses (see Figure 1). And, as it turns out, there are even a few pairs of matter and antimatter. It is shown further that there actually four pairs rather than two as in Figure 1.

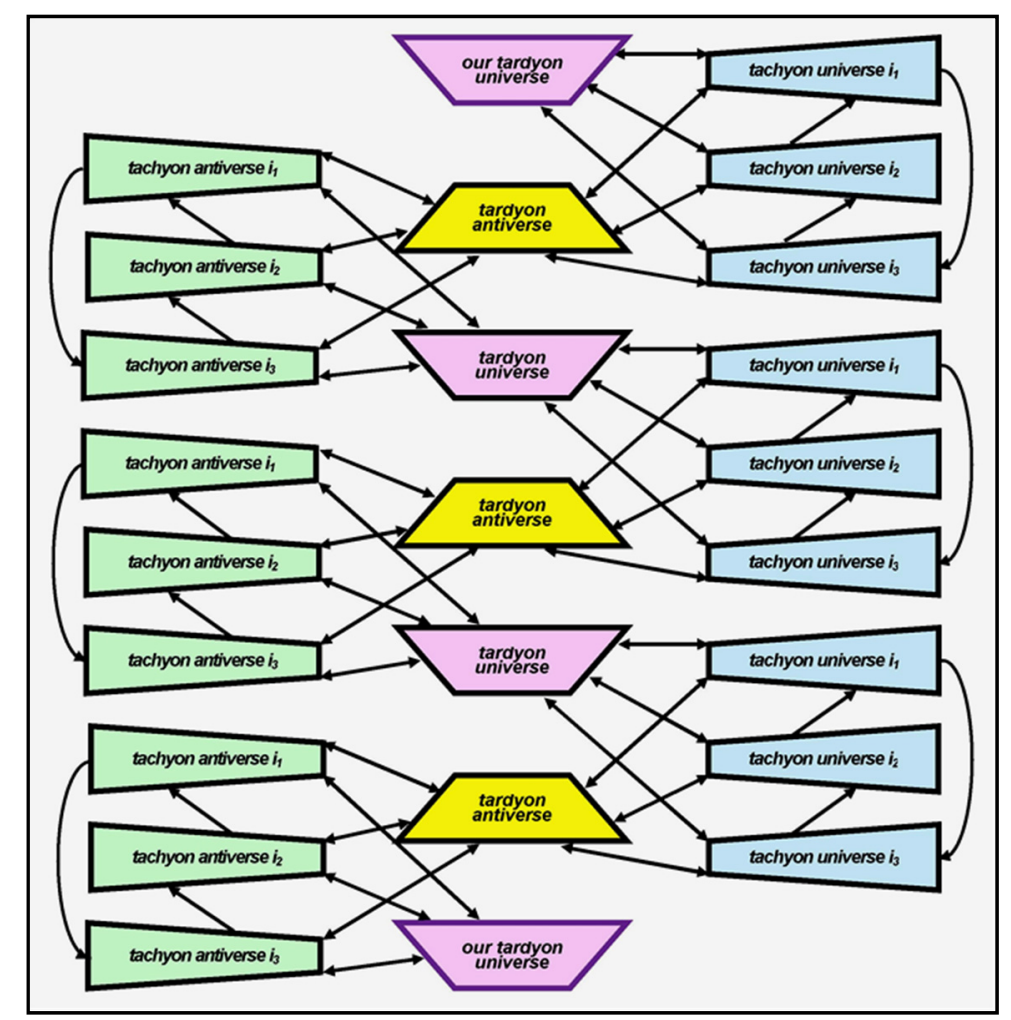

Figure 2. Possible structure of the hidden Multiverse, corresponding to the principle of physical reality of quaternions 


\section{Adjustment of the Structure of the Hidden Multiverse Pursuant to the WMAP and Planck Data}

Astrophysical research performed in recent years using WMAP (Adam et al., 2015) and Planck (Adam et al., 2015) spacecrafts that allowed receiving extremely valuable results is of great interest. According to the data obtained by the Planck spacecraft, the total mass-energy of the whole Multiverse consists of $4.9 \%$ ordinary (baryonic) matter (earlier WMAP estimate - 4.6\%), 26.8\% dark matter (according to WMAP - 22.4\%) and 68.3\% dark energy (according to WMAP - 73\%). Therefore, considering mass-energy of each universe in the hidden Multiverse to be approximately equal to our visible tardyon universe, it can be concluded that:

- $\quad$ according to Planck data, the whole hidden Multiverse consists of $100 \% / 4,9 \%=20,4$ parallel universes (according to WMAP data it consists of $100 \% / 4,6 \%=21,8$ parallel universes);

- according to Planck data, dark matter consists of $26,8 \% / 4,9 \%=5,5$ parallel universes (according to WMAP data it consists of $22,4 \% / 4,6 \%=4,9$ parallel universes);

- according to Planck data, dark energy consists of $68,3 \% / 4,9 \%=13,9$ parallel universes (according to WMAP data it consists of $73,0 \% / 4,6 \%=15,9$ parallel universes);

In fact, the number of universes shall actually be an integer. The difference between the results obtained and integers can be explained both by measurement errors and some diversity of mass-energies of different universes. Therefore, given these circumstances, it can be assumed that the hidden Multiverse contains 20 ... 22 universes, of which one universe is ours; $5 . . .6$ adjacent universes constitute dark matter and $14 \ldots 16$ of the rest of the universes constitute dark energy.

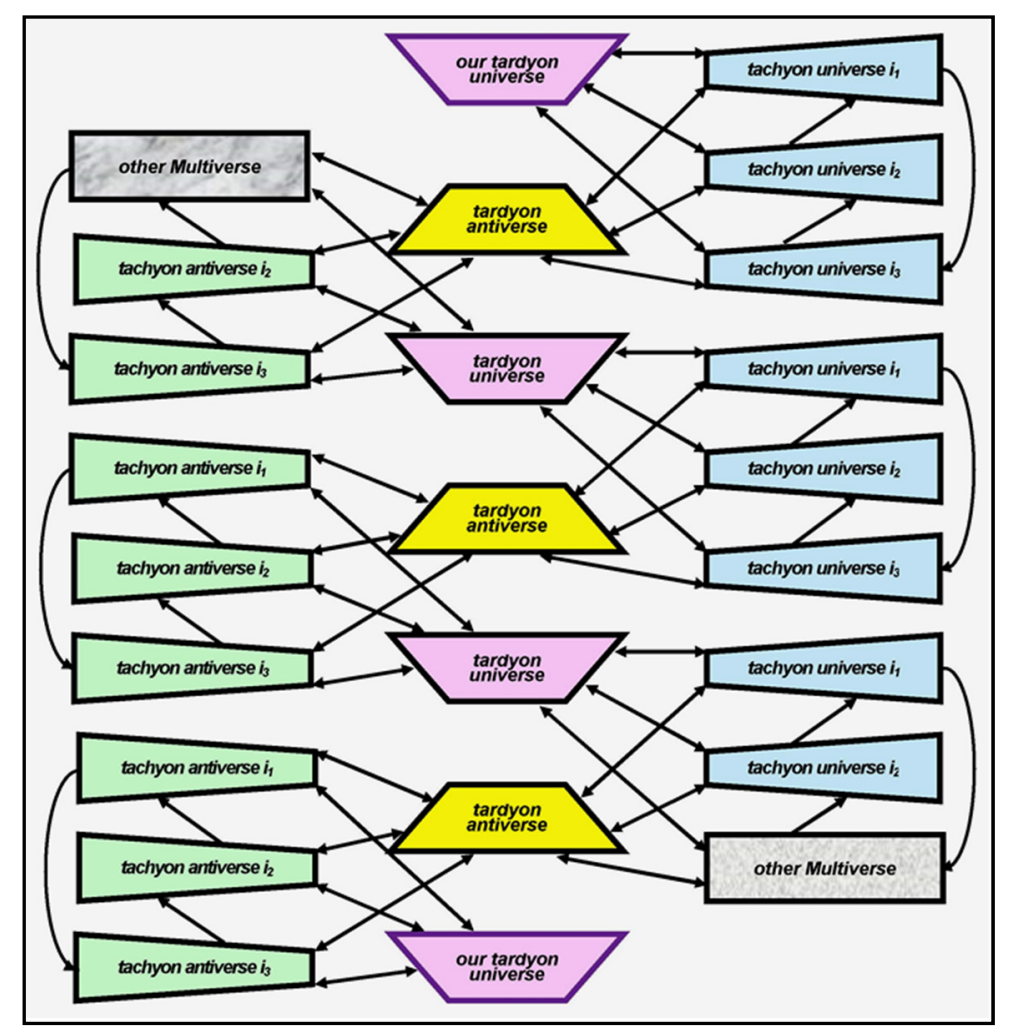

Figure 3. One of the most probable structures of the hidden Multiverse corresponding to the principle of physical reality of quaternions

However..., these results correspond neither to formulas (3) and (4) nor to the structure of the hidden Multiverse, shown in Figure 1. Indeed, Figure 1 shows that our tardyon universe has only two adjacent universes, rather than 5 ... 6 .

Such a result actually has an explanation. It is as follows. The structure of the hidden Multiverse shown in Figure 1 corresponds to the principle of physical reality of complex numbers, as relativistic formulas (3) and (4) contain only one imaginary unit. It means that one tachyon universe and one tachyon antiverse are adjacent to our 
tardyon universe. However, according to the WMAP and Planck data our universe should be allegedly adjacent to six universes, i.e. three tachyon universes and three tachyon antiverses. Hence, the relevant relativistic formulas should contain three imaginary units. In accordance with this logic, the structure of the hidden Multiverse corresponding to the WMAP and Planck data might have quaternion structure, as exactly quaternions $\sigma+i_{1} \omega_{1}+i_{2} \omega_{2}+i_{3} \omega_{3}$ comprise three imaginary units $i_{1}, i_{2}, i_{3}$, connected by relations (Kantor \& Solodovnikov, 1989)

$$
\begin{gathered}
i_{1}^{2}=i_{2}^{2}=i_{3}^{2}=-1 \\
i_{1} i_{2} i_{3}=i_{2} i_{3} i_{1}=i_{3} i_{1} i_{2}=-1 \\
i_{1} i_{3} i_{2}=i_{2} i_{1} i_{3}=i_{3} i_{2} i_{1}=1
\end{gathered}
$$

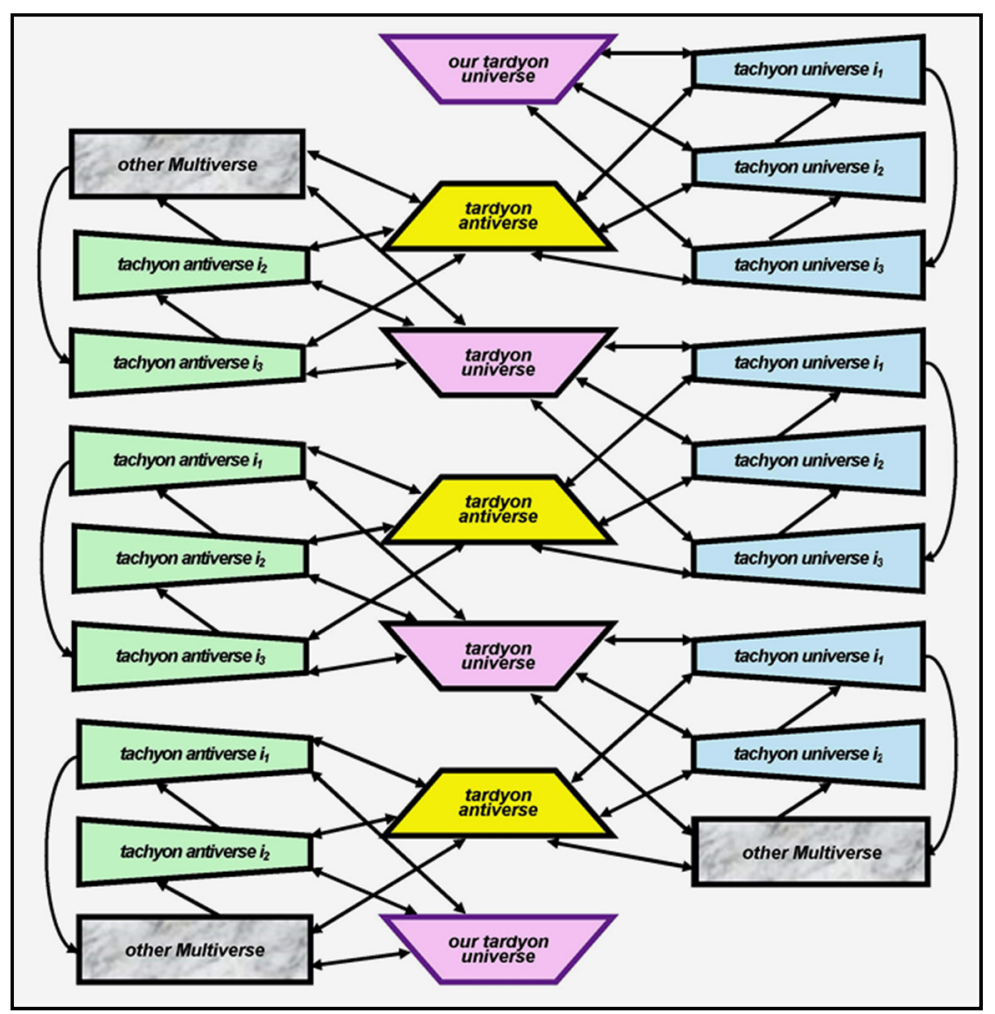

Figure 4. Another most probable structure of the hidden Multiverse corresponding to the principle of physical reality of quaternions

Therefore, the formulas (1) and (2) should be adjusted once more in the following way

$$
\begin{aligned}
& m=\frac{m_{0}\left(i_{1}\right)^{q}\left(i_{2}\right)^{r}\left(i_{3}\right)^{s}}{\sqrt{1-[v / c-(q+r+s)]^{2}}}=\frac{m_{0}\left(i_{1}\right)^{q}\left(i_{2}\right)^{r}\left(i_{3}\right)^{s}}{\sqrt{1-(w / c)^{2}}} \\
& \Delta t=\Delta t_{0}\left(i_{1}\right)^{q}\left(i_{2}\right)^{r}\left(i_{3}\right)^{s} \sqrt{1-[v / c-(q+r+s)]^{2}}= \\
& =\Delta t_{0}\left(i_{1}\right)^{q}\left(i_{2}\right)^{r}\left(i_{3}\right)^{s} \sqrt{1-(w / c)^{2}}
\end{aligned}
$$

where $\boldsymbol{q}$ is the total number of parallel universes, penetration into which was made through portals, corresponding to the imaginary unit $\boldsymbol{i}_{\boldsymbol{1}}$, with increasing distance from our tardyon universe;

$r$ is the total number of parallel universes, penetration into which was made through portals, corresponding to the imaginary unit $\boldsymbol{i}_{2}$, with increasing distance from our tardyon universe; 
$\boldsymbol{s}$ is the total number of parallel universes, penetration into which was made through portals, corresponding to the imaginary unit $\boldsymbol{i}_{3}$, with increasing distance from our tardyon universe;

$\boldsymbol{v}$ is the velocity measured from our tardyon universe, which, therefore, can be called tardyon velocity;

$c$ is the speed of light;

$\boldsymbol{w}=\boldsymbol{v}-(\boldsymbol{q}+\boldsymbol{r}+\boldsymbol{s}) \boldsymbol{c}$ is the local velocity for corresponding universe, which can take values only in the range $i_{3} i_{1}=i_{2}$.

Other relativistic formulas of the STR can be adjusted in a similar manner.

One of the quaternion structures of the hidden Multiverse corresponding to the formulas (6) and (7) is shown in Figure 2. As seen, in the structure of the Multiverse that looks like a screw collar, unlike in the structure shown in Figure 1, our tardyon universe has just six adjacent universes, three of which are tachyon universes and another three are tachyon antiverses. However, in addition to bidirectional portals which correspond to the relation (5a) and are denoted by bidirectional arrows, the hidden Multiverse can also contain unidirectional portals which correspond to the relations $(5 \mathrm{~b}, \mathrm{c})$ and are denoted by bidirectional arrows. The unidirectional portals are dangerous, because they don't allow returning back to the home universe, once you get into them. Figure 2 shows our tardyon universe twice, as it is both conditional beginning and end of reference of parallel universes in a closed structure of the hidden Multiverse.

However, the structure given in Figure 2 is still not completely in line with the data of WMAP and Planck space stations, since it contains 24 universes, rather than $20 \ldots 22$. Consequently, the closed structure of the hidden Multiverse should be adjusted again, i.e., 2 ... 4 tachyon universes and/or antiverses should be excluded from it. In this case the hidden Multiverse would have edges. This circumstance gives rise to the inevitable question: What is behind the edges? Notably, we have no experimental data suggesting an answer to this question. Therefore, we can only assume. The most likely would be the assumption that there are other Multiverses behind the edges of our hidden Multiverse, i.e. there are not only Multiverses, but also Hyperverses. Or, perhaps, even larger structures. Moreover, other Multiverses adjacent to our hidden Multiverse are unobservable to us both by electromagnetic and gravitational manifestations.

Figure 3 shows an example of a probable structure of the hidden Multiverse containing 22 universes, in which our tardyon universe is adjacent to six tachyon universes and antiverses. Figure 4 shows another example of a probable structure of the hidden Multiverse containing 21 universes, in which our tardyon universe is adjacent to five tachyon universes and antiverses. There may be also other structures of the hidden Multiverse. This fact may be another reason that according to the WMAP and Planck data the number of universes corresponding to dark matter is $5 \ldots 6$.

\section{Verifiability of the Hypothesis of the Hidden Multiverse}

A great number of interesting Multiverse hypotheses have been proposed by now (Deutch, 1998; 2012; Greene, 2000; 2011; Vilenkin, 2006; Steinhardt \& Turok, 2007; Weinberg, 2008; Carr, 2009; Tegmark, 2015). However, they would get experimental confirmation neither now nor in the distant future. They neither could be refuted. That is to say, they are unverifiable. Or, in other words, they are virtually non-existent for us.

In contrast, since the hypothesis of the hidden Multiverse is based on the experimental data rather than on postulates, it is verifiable and its actual physical existence is experimentally confirmed. In fact:

- Firstly, the phenomenon of dark matter and dark energy explained above can be mentioned as an experimental proof of its existence.

- Secondly, some results of experiments at the Large Hadron Collider and other accelerators can serve as evidence of its existence. The experiments resulted in mass defect, i.e., total beam mass before acceleration turned out to be greater than total beam mass in the experiment. This result can be explained by formation of tachyons that disappeared in a tachyon universe or antiverse. This process could take place, for example, as a result of the creation of short-time micro or mini portals, like formation of macro portal in the episode with the Eldridge destroyer.

- Finally, discovery of portals on the Earth, which are anomalous zones, can also be one more proof (Antonov, 2015). 


\section{Conclusion}

The hypothesis of the hidden Multiverse described in the article has every reason to be called a theory. The theory, in contrast to numerous unverifiable Multiverse hypotheses, is built on a solid foundation based on the general scientific principle of the physical reality of imaginary, complex and hyper complex numbers proved by the author.

This principle allowed us to reveal fundamental errors in the current version of the STR and correct them. To the credit of Albert Einstein it should be noted that he didn't exclude such an adjustment of the STR in the future. He wrote: "No single idea, which I would be sure that it will stand the test of time."

The theory of the hidden Multiverse developed on the basis of the adjusted version of the STR has explained its structure, the phenomenon of dark matter and dark energy, where antimatter is and what the portals are, as well as solved a number of other issues of modern astrophysics.

\section{References}

Adam, R., Ade, P. A. R., Aghanim, N., Akrami, Y., Alves, M. I. R., Argüeso, F., ... \& Baccigalupi, C. (2016). Planck 2015 results-I. Overview of products and scientific results. Astronomy \& Astrophysics, 594, A1. http://doi.org/ 10.1051/0004-6361/201527101

Adam, T., Agafonova, N., Aleksandrov, A., Altinok, O., Sanchez, P. A., Anokhina, A., ... \& Badertscher, A. (2012). Measurement of the neutrino velocity with the OPERA detector in the CNGS beam. Journal of High Energy Physics, 2012(10), 1-37. http://doi.org/ 10.1007/JHEP10(2012)093

Adamson, P., Andreopoulos, C., Arms, K. E., Armstrong, R., Auty, D. J., Avvakumov, S., ... \& Barr, G. (2007). Measurement of neutrino velocity with the MINOS detectors and NuMI neutrino beam. Physical Review D, 76(7), 072005. http://doi.org/ 10.1103/PhysRevD.76.072005

Alfvén, H. (1966). Worlds-Antiworlds: Antimatter in Cosmology. San Francisco: W. H. Freeman \& Co.

Antonello, M., Aprili, P., Baiboussinov, B., Ceolin, M. B., Benetti, P., Calligarich, E., ... \& Cline, D. B. (2012). Measurement of the neutrino velocity with the ICARUS detector at the CNGS beam. Physics Letters B, 713(1), 17-22. http://doi.org/ 10.1016/j.physletb.2012.05.033

Antonov, A. A. (2008). Physical Reality of Resonance on Complex Frequencies. European Journal of Scientific Research, 21(4), 627-641.

Antonov, A. A. (2009). Resonance on Real and Complex Frequencies. European Journal of Scientific Research, 28(2), 193-204.

Antonov, A. A. (2010a). New Interpretation of Resonance. International Journal of Pure and Applied Sciences and Technology, 1(2), 1-12. http://doi.org/10.17686/sced_rusnauka_2010-888

Antonov, A. A. (2010b). Oscillation processes as a tool of physics cognition. American Journal of Scientific and Industrial Research, 1(2), 342-349. http://doi.org/ 10.5251/ajsir.2010.1.2.342.349

Antonov, A. A. (2010c). Solution of algebraic quadratic equations taking into account transitional processes in oscillation systems. General Mathematics $\quad$ Notes, $\quad 1(2), \quad 11-16$. http://doi.org/10.17686/sced_rusnauka2010-887

Antonov, A. A. (2011). Structure of the Multiverse. British Journal of Science, 2(2) 51-60. http://doi.org/10. 17686/sced_rusnauka_2011-892

Antonov, A. A. (2012). Earth, portals, parallel universes. American Journal of Scientific and Industrial Research, 3(6), 464-473. http://doi.org/ 10.5251/ajsir.2012.3.6.464.473

Antonov, A. A. (2014a). Correction of the special theory of relativity: physical reality and nature of imaginary and complex numbers. American Journal of Scientific and Industrial Research, 5(2), 40-52. http://doi.org/ 10.5251/ ajsir.2014.5.2.40.52.

Antonov, A. A. (2014b). Verification of the second postulate of the special relativity theory. Global Journal of Science Frontier Research: A Physics \& Space Science, 14(3), 51-59. Retrieved from http://globaljournals.org.GJSFR_Volume 14/7-Verification-of-the- Second-Postulate.pdf

Antonov, A. A. (2015a). Ohm's Law explains astrophysical phenomenon of dark matter and dark energy. Global Journal of Physics, 2(2), 145-149. Retrieved from http://gpcpublishing.com/index.php?journal=gjp\&page= article\&op= view\&path\%5B\%5D\%-294 
Antonov, A. A. (2015b). Adjustment of the special theory of relativity according to the Ohm's law. American Journal of Electrical and Electronics Engineeing, 3(5), 124-129. http://doi.org/ 10.12691/ajeee-3-5-3

Antonov, A. A. (2015c). Physical reality of complex numbers is proved by research of resonance. General Mathematics Notes, 31(2), 34-53. Retrieved from http://www.emis.de/journals/GMN/yahoo_site_admin/ assets/docs/4_GMN-9212-V31N2.1293701.pdf

Antonov, A. A. (2015d). Why Dark Matter and Dark Energy are Invisible. Optics, 4(6), 43-47. http://doi.org/ 10.11648/j. optics.20150406.12

Antonov, A. A. (2015e). Hidden Multiverse. International Journal of Advanced Research in Physical Science, 2(1), 25-32. http://doi.org/10.17686/sced_rusnauka_2015-903

Antonov, A. A. (2015f). Hidden Multiverse: Explanation of Dark Matter and Dark Energy Phenomena. International Journal of Physics, 3(2) 84-87. http://doi.org/ 10.12961/ijp-3-2-6

Antonov, A. A. (2015g). Hidden Multiverse: explanation of dark matter and dark energy. Cosmology, 19, 40-61. Retrieved from http://cosmology.com/Aliens1.html

Antonov, A. A. (2015h). Principles and structure of the real Multiverse: explanation of dark matter and dark energy phenomena. American Journal of Modern Physics, 4(1), 1-9. http://doi.org/ 10.11648/j.ajmp.20150401.11

Antonov, A. A. (2015i). Explanation of dark matter and dark energy phenomena. Global Journal of Science Frontier Research: A Physics and Space Science, 15(1), 33-38. http://doi.org/10.17686/sced_rusnauka_ 2015-902

Antonov, A. A. (2015k). The astrophysical phenomenon of dark matter and dark energy proves the existence of the hidden Multiverse. American Journal of Modern Physics, 4(4), 180-188. http://doi.org/ 10.11648/j.jamp. 20150404.14

Antonov, A. A. (20151). Where to look for alien civilisations. Cosmology. Commentaries: Stephen Hawking's Aliens. The Search for Intelligent Extraterrestrial Life. Project Breakthrough Listen. Retrieved from http://cosmology.com/Aliens1.html

Antonov, A. A. (2016a). Ohm's law is the general law of exact sciences. Ponte, 72(7), 131-142. http://doi.org/ $10.21506 /$ j. ponte. $2016.7 / 9$

Antonov, A. A. (2016b). Hypothesis of the hidden multiverse explains dark matter and dark energy. Journal of Modern Physics, 7, 1228-1246. http://doi.org/ 10.4236/jmp.2016.710111

Antonov, A. A. (2016c). Physical Reality and Nature of Imaginary, Complex and Hypercomplex Numbers. General Mathematics Notes, 35(2), 40-63. Retrieved from http://www.geman.in/yahoo_site_admin/assets/ docx/4_GMN- 10932-V35N2.31895146.pdf

Antonov, A. A., \& Bazhev, V. M. (1970). Means of rising deflecting currents for spiral beam sweep on the CRT screen. Patent of USSR \# 433650.

Antonov, A. A. (1987). Investigation of resonance. Preprint \# 67. G.E. Pukhov Institute for Modelling in Energy Engineerig. Ukrainian Academy of Sciences. Kiev.

Carr, B. (ed). (2009). Universe or Multiverse? Cambridge: Cambridge Univ. Press.

Deutch, D. (1998). The Fabric of Reality: The Science of Parallel Universes and Its Implications. NY: Penguin Books.

Deutsch, D. (2012). The Beginning of Infinity: Explanations That Transform the World. Reprint Edition. NY: Penguin Books.

Einstein, A. (1920). Relativity: The Special and General Theory. NY: H. Holt and company.

Feinberg, G. (1967). Possibility of faster-than-light particles. Physical Review, 159(5), 1089-1105. http://doi.org/ 10.1103/ PhysRev.159.1089

Frazer, G. (2004). Antimatter: The Ultimate Mirror. Cambridge: Cambridge University Press.

Freeman, K., \& McNamara, G. (2006). In Search of Dark Matter. NY: Springer.

Frova, A., \& Marenzana, M. (2006). Thus spoke Galileo: The great scientist's ideas and their relevance to the present day. NY: Oxford University Press, 
Greene, B. (2000). The Elegant Universe: Superstrings, Hidden Dimensions, and the Quest for the Ultimate Theory. NY: Random House Inc.

Greene, B. (2011). The Hidden Reality: Parallel Universes and the Deep Laws of the Cosmos. NY: Random House Inc.

Hawking, S. W., \& Penrose, R. (2010). The Nature of Space and Time. Princeton University Press.

Hinshaw, G., Larson, D., Komatsu, E., Spergel, D. N., Bennett, C. L., Dunkley, J., ... \& Page, L. (2013). Nine-year Wilkinson Microwave Anisotropy Probe (WMAP) observations: cosmological parameter results. The Astrophysical Journal Supplement Series, 208(2), 19. http://doi.org/ 10.1088/0067 -0049/208/2/19

Kantor, I. L., \& Solodovnikov, A. S. (1989). Hypercomplex numbers. Berlin: Springer Verlag.

Lyahov, V. V., \& Nechshadim, V. M. (12 Mar 2001). Complex numbers and physical reality. http://arxiv.org/abs/physics/0102047

Nicolson, I. (2007). Dark Side of the Universe: Dark Matter, Dark Energy, and the Fate of the Cosmos. Baltimore: Johns Hopkins University Press.

Ohm, G. S. (2014). Die galvanische Kette. Göttingen: Verlag Der Wissenschaften. Neumünster Neumünster Neumünster.

Spenkov, G. P. (2014). Physical meaning of imaginary unit “i”. Encyclopedia of Russian Thought: Reports to Russian Physical Society, 20, 70- 81. Retrieved from http://shpenkov.janmax.com/ImaginUnitRus.pdf

Steinhardt, P. J., \& Turok, N. (2007). Endless Universe: Beyond the Big Bang. NY: Doubleday.

Steinmetz, C. P. (2010). Theory and Calculation of Electric Circuit. Charlstone, SC Nabu Press.

Tanaka, S. (1960). Theory of matter with superlight velocity. Progress of Teoretical Physics (Kyoto), 24(1), 171-200. http://sciepub.com/reference/136329

Tegmark, M. (2015). Our Mathematical Universe: My Quest for the Ultimate Nature of Reality. NY: Vintage.

Vilenkin, A. (2006). Many Worlds in One: The Search for Other Universes. NY: Hill and Wong.

Weinberg, S. (2008). Cosmology. Oxford: Oxford University Press.

\section{Copyrights}

Copyright for this article is retained by the author(s), with first publication rights granted to the journal.

This is an open-access article distributed under the terms and conditions of the Creative Commons Attribution license (http://creativecommons.org/licenses/by/4.0/). 